21st Particles and Nuclei International Conference (PANIC 2017)

International Journal of Modern Physics: Conference Series

Vol. 46 (2018) 1860016 (6 pages)

(C) The Author(s)

DOI: $10.1142 / \mathrm{S} 2010194518600169$

\title{
Measurements of Hadron Resonance Production with ALICE
}

\author{
Enrico Fragiacomo (on behalf of the ALICE Collaboration) \\ Istituto Nazionale di Fisica Nucleare, Sezione di Trieste \\ via Valerio 2, Trieste 34126, Italy \\ enrico.fragiacomo@ts.infn.it
}

Published 3 May 2018

\begin{abstract}
Short-lived resonances have long been recognized as good probes to investigate the latestage evolution of ultra-relativistic heavy-ion collisions. More recently, resonances with different masses and quark composition have provided valuable contributions to the understanding of strangeness production, in particular for the observed multiplicitydependent enhancement in small systems. In this light, a comprehensive review is presented of the most recent ALICE results on $\rho(770)^{0}, K^{*}(892)^{0}, \phi(1020), \Sigma(1385)^{ \pm}$, $\Lambda(1520)$ and $\Xi(1530)^{0}$ production in $\mathrm{pp}, \mathrm{p}-\mathrm{Pb}$ and $\mathrm{Pb}-\mathrm{Pb}$ collisions at various collision energies at the LHC, including results from the latest $\mathrm{Pb}-\mathrm{Pb}$ run at $\sqrt{s_{\mathrm{NN}}}=5.02 \mathrm{TeV}$.
\end{abstract}

Keywords: Relativistic heavy-ion collisions; late-stage evolution; hadronic resonances.

\section{Introduction}

The production yields of hadrons measured in relativistic heavy-ions collisions are described by fits of statistical hadronization models (SHM) ${ }^{1}$ with few parameters such as temperature, volume and baryo-chemical potential at the chemical freezeout. Differences to model predictions are given by hadron resonances whose yields in central collisions appear suppressed, likely due to re-scattering ${ }^{2}$ of the decay products in the last stage of the collision before thermal decoupling (kinetic freeze-out). Suppression of hadron resonances has been early found by the NA49 collaboration for $\mathrm{K}^{*}(892)^{4}$ and $\Lambda(1520)^{5}$ and by the STAR collaboration for $\mathrm{K}^{*}(892)^{6}$. The ALICE collaboration has studied a comprehensive set of hadron resonances, $\rho(770)^{0}, \mathrm{~K}^{*}(892)^{0}$ and $\phi(1020)^{7,8,9}, \Sigma(1385)^{ \pm}$and $\Xi(1530)^{0}{ }^{10,11}$, and $\Lambda(1520)$, for different energies and colliding systems, showing a clear suppression for $\rho^{0}, \mathrm{~K}^{* 0}$ and $\Lambda^{* 0}$ in central $\mathrm{Pb}-\mathrm{Pb}$ collisions. Strangeness production is discussed in Section 2 as baseline for the study of strange resonances. The ratios of resonance yields to stable-particle yields as a function of multiplicity are presented in Section 3 and discussed in the light of re-scattering effects. A detailed description of the ALICE apparatus and of its performance can be found in Ref. 3

This is an Open Access article published by World Scientific Publishing Company. It is distributed under the terms of the Creative Commons Attribution 4.0 (CC-BY) License. Further distribution of this work is permitted, provided the original work is properly cited. 


\section{E. Fragiacomo}

\section{Strangeness production}

The ratios of $p_{\mathrm{T}}$-integrated yields to the pion yields as a function of the charged particle multiplicity at mid-rapidity are shown in Fig. 1 for kaons and hyperons. New results from $\mathrm{Pb}-\mathrm{Pb}$ collisions at $\sqrt{s_{\mathrm{NN}}}=5.02 \mathrm{TeV}$ are presented. Charged particle multiplicity is related to the number of participants in the collision and to the size of the produced volume. An increase from low-multiplicity pp to central $\mathrm{Pb}-\mathrm{Pb}$ collisions is observed for both the kaon-to-pion ratio and the hyperon-to-pion ratios.

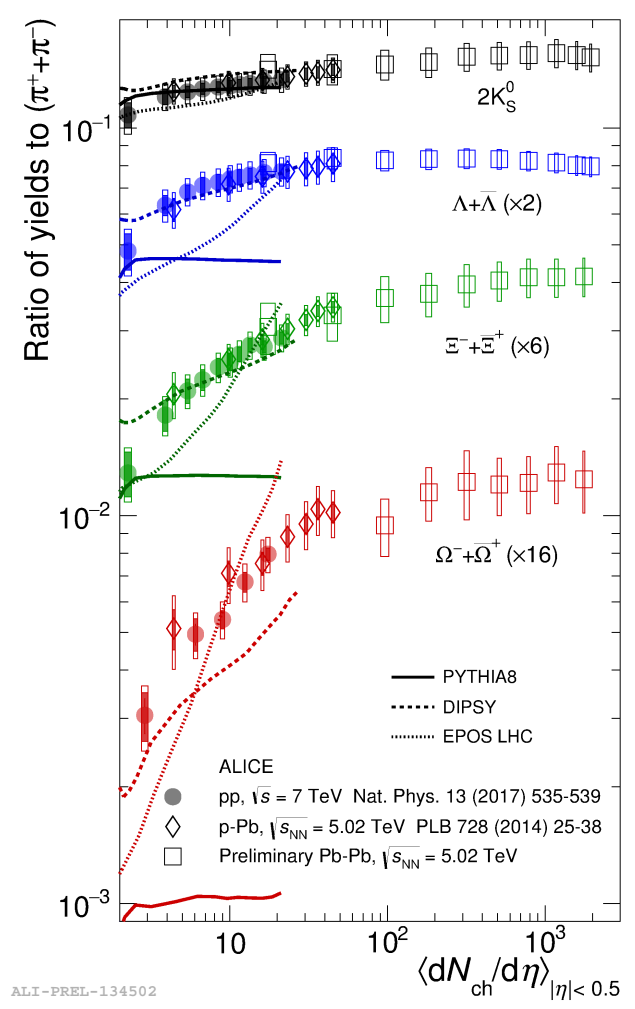

Fig. 1. Ratios of $p_{\mathrm{T}}$-integrated yields to the pion yield for kaons and hyperons as a function of the charged particle multiplicity at mid-rapidity.

The enhancement of strangeness production in heavy-ion collisions, historically proposed as a signature of quark-gluon plasma formation ${ }^{12}$, is explained in the context of SHM as the canonical suppression of strangeness production in small systems as a consequence of local strangeness conservation ${ }^{13}$. It remains under debate whether a dynamical equilibration takes place or other processes at hadronization account for the strangeness saturation observed in $\mathrm{Pb}-\mathrm{Pb}$ collisions ${ }^{14}$. The novelty in Fig. 1 is given by the increase of the kaon- and hyperon-to-pion ratios as 
a function of multiplicity for pp collisions, following the trend observed for $\mathrm{p}-\mathrm{Pb}$ collisions.

Commonly used Monte Carlo models such as PYTHIA8 ${ }^{16}$ are unable to reproduce the observed increasing trend, while mechanisms such as the core-corona interplay (implemented in EPOS ${ }^{18}$ ) and color-rope (DIPSY ${ }^{19}$ ) seem to describe qualitatively, but not yet satisfactorily from a quantitative point of view, the multiplicity dependence of the results. Also, the amount of the increase is found to depend on the strangeness content of the hadron and not on its mass, as demonstrated by the flatness of the $\Sigma^{* \pm} / \Lambda$ and $\Xi^{* 0} / \Xi$ ratios, shown in Fig. 2 for minimum-bias pp and $\mathrm{p}-\mathrm{Pb}$ collisions. The results for $\Sigma^{* \pm} / \Lambda$ are in good agreement with the results


Fig. 2. (Left) Ratio of $\Sigma^{* \pm}$ to $\Lambda$ and (Right) ratio of $\Xi^{* 0}$ to $\Xi^{-}$measured in pp , d-Au and $\mathrm{p}-\mathrm{Pb}$ collisions, as a function of the charged particle multiplicity at mid-rapidity.

from STAR ${ }^{20,23}$ and common Monte Carlo generators (PYTHIA8 ${ }^{16}$, DPMJET ${ }^{17}$ ), while for $\Xi^{* 0} / \Xi$ the agreement is not completely satisfactory. Both ratios in $\mathrm{p}-\mathrm{Pb}$ agree with SHM predictions ${ }^{1,15}$ for $\mathrm{Pb}-\mathrm{Pb}$ collisions.

\section{Late stage of the collision}

The considerations in Section 2 suggest to study as a function of the system size the ratios of resonance yields to the yields of stable hadrons with same strangeness content and look for deviations from a flat behavior. Figure 3 shows the ratios $\rho / \pi, \mathrm{K}^{*} / \mathrm{K}, \phi / \mathrm{K}, \Lambda^{*} / \Lambda$ and $\Xi^{*} / \Xi$ as a function of the cubic root of the charged particle multiplicity at mid-rapidity, which can be used as a proxy for the system size ${ }^{26}$. A suppression at high multiplicities is expected, whose magnitude partly depends on the lifetime of the resonance compared to the lifetime of the hadronic fireball ${ }^{2}$. The shortest-lived resonances, having a larger probability to decay in the hadronic fireball, should tend to be more suppressed, unless regeneration effects play a role to contrast this behaviour. This is indeed seen in Fig. 3a-b for both $\rho$ (lifetime $\tau=1.3 \mathrm{fm} / c^{27}$ ) and $\mathrm{K}^{* 0}\left(\tau=4.2 \mathrm{fm} / c^{27}\right)$, whose ratios to long-lived particles in central $\mathrm{Pb}-\mathrm{Pb}$ collisions are smaller by $\sim 40 \%$ with respect to the same 


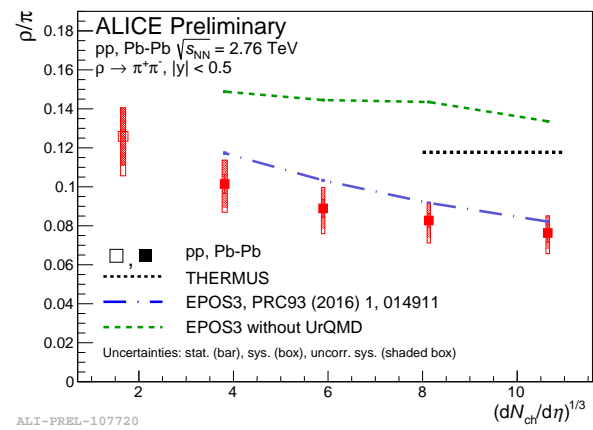

(a)

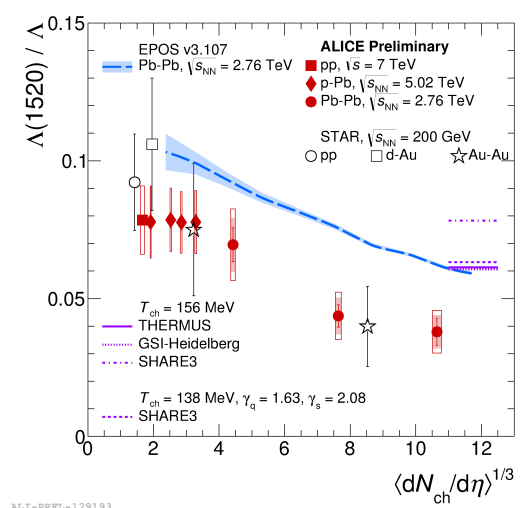

(c)

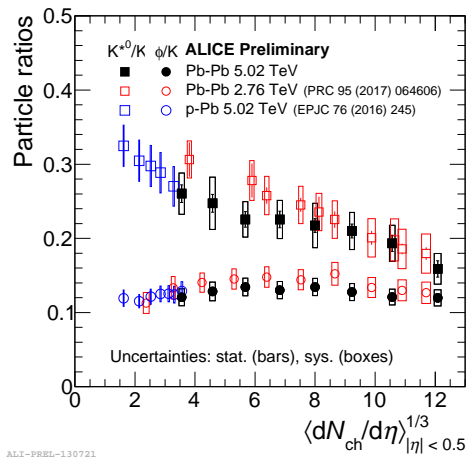

(b)

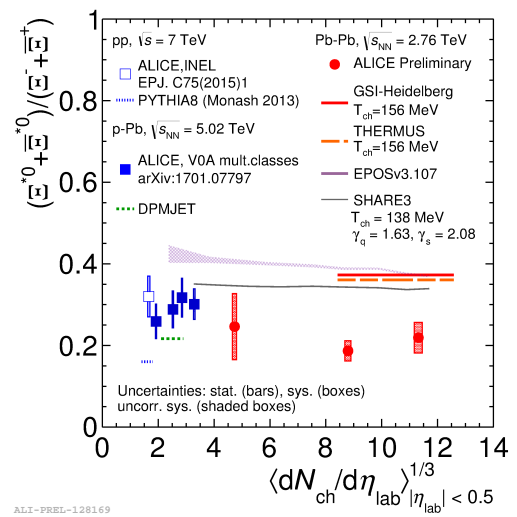

(d)

Fig. 3. Yield ratios of resonances to stable hadrons with same strangeness content.

ratios in small systems. Conversely, for $\phi$, whose lifetime $\left(\tau=46.2 \mathrm{fm} / c^{27}\right)$ is an order of magnitude longer than the lifetime of $\mathrm{K}^{* 0}$, the ratio to kaons in Fig. $3 \mathrm{~b}$ does not seem to deviate from a flat behavior, suggesting that $\phi$ survives unaffected through the hadronic medium. The newest results on $\mathrm{K}^{* 0} / \mathrm{K}$ and $\phi / \mathrm{K}$ in $\mathrm{Pb}-\mathrm{Pb}$ collisions at $\sqrt{s_{\mathrm{NN}}}=5.02 \mathrm{TeV}$ confirm the trends observed at $2.76 \mathrm{TeV}$. Concerning baryons, a relatively large suppression is seen for $\Lambda^{*}\left(\tau=12.6 \mathrm{fm} / c^{27}\right)$, while $\Xi^{* 0}$ $\left(\tau=21.7 \mathrm{fm} / c^{27}\right)$ seems only weakly affected by re-scattering, maybe due to its larger lifetime, but more precise measurements are needed in order to draw stronger conclusions.

All ratios, except $\phi / \mathrm{K}$, in central $\mathrm{Pb}-\mathrm{Pb}$ collisions are lower than the thermal model predictions (shown for $\rho / \pi, \Lambda^{*} / \Lambda$ and $\Xi^{*} / \Xi$ in Fig. 3). The $\Lambda^{*} / \Lambda$ ratio in Fig. $3 \mathrm{c}$ is also compared to STAR measurements in pp, $\mathrm{d}-\mathrm{Au}$ and $\mathrm{Au}-\mathrm{Au}$ collisions. The $\Lambda^{*} / \Lambda$ results obtained in $\mathrm{Pb}-\mathrm{Pb}$ at $\sqrt{s_{\mathrm{NN}}}=2.76 \mathrm{TeV}$ follow the trend of the results from the STAR experiment, with smaller uncertainties.

Regeneration processes competing with re-scattering can increase the yields of resonances ${ }^{28}$. EPOS3 with UrQMD ${ }^{29}$, which includes a modeling of both 
re-scattering and regeneration, well describes the suppression trend, as seen for $\rho / \pi$ in Fig. 3a and $\Lambda^{*} / \Lambda$ in Fig. 3c, while the prediction without UrQMD does not reproduce the decreasing trend, shown for $\rho / \pi$ in Fig. 3a.

\section{Conclusions}

Re-scattering of decay products prevent the reconstruction of the shortest-lived hadronic resonances $\left(\rho, \mathrm{K}^{* 0}\right.$ and $\left.\Lambda^{*}\right)$ decaying during the late hadronic stage of central collisions. The effect is seen in the deviation from the expected flat behavior of the ratios of resonance yields to the yields of stable particles with the same strangeness content, when it is studied as a function of the system size. The effect is not observed for the longest-lived resonances $\left(\phi\right.$ and $\left.\Xi^{* 0}\right)$. Transport models are successfully coupled to event generators to gain information on the late-stage evolution of the collision.

\section{References}

1. A. Andronic, P. Braun-Munzinger, and J. Stachel, "Thermal hadron production in relativistic nuclear collisions: The hadron mass spectrum, the horn, and the QCD phase transition," Phys. Lett. B673 (2009) 142-145, arXiv:0812.1186 [nucl-th].

2. G. Torrieri and J. Rafelski, "Strange hadron resonances as a signature of freeze-out dynamics," Phys. Lett. B509 (2001) 239-245, hep-ph/0103149.

3. ALICE Collaboration, B. Abelev et al., "Performance of the ALICE Experiment at the CERN LHC," Int. J. Mod. Phys. A29 (2014) 1430044, arXiv: 1402.4476 [nucl-ex].

4. NA49 Collaboration, T. Anticic et al., " $\mathrm{K}(892)^{0}$ and $\overline{\mathrm{K}}(892)^{0}$ production in central $\mathrm{Pb}+\mathrm{Pb}, \mathrm{Si}+\mathrm{Si}, \mathrm{C}+\mathrm{C}$, and inelastic $\mathrm{p}+\mathrm{p}$ collisions at $158 \mathrm{~A} \mathrm{GeV",} \mathrm{Phys.} \mathrm{Rev.}$ C 84, 064909 (2011)

5. NA49 Collaboration, V. Friese et al., "Production of Strange Resonances in $\mathrm{C}+\mathrm{C}$ and $\mathrm{Pb}+\mathrm{Pb}$ Collisions at $158 \mathrm{AGeV}$ ", Nucl. Phys. A698 (2002) 487c-490c

6. STAR Collaboration, M.M. Aggarwal et al., " $\mathrm{K}^{0}$ production in $\mathrm{Cu}+\mathrm{Cu}$ and $\mathrm{Au}+\mathrm{Au}$ collisions at $\sqrt{s_{\mathrm{NN}}}=62.4 \mathrm{GeV}$ and $200 \mathrm{GeV}$ ", Phys. Rev. C 84, 034909 (2011)

7. ALICE Collaboration, B. Abelev et al., "Production of $\mathrm{K}^{*}(892)^{0}$ and $\phi(1020)$ in pp collisions at $\sqrt{s_{\mathrm{NN}}}=7 \mathrm{TeV}$," Eur. Phys. J. C72 (2012) 2183, arXiv:1208.5717 [nucl-ex].

8. ALICE Collaboration, J. Adam et al., "Production of $\mathrm{K}^{*}(892)^{0}$ and $\phi(1020)$ in $\mathrm{p}-\mathrm{Pb}$ collisions at $\sqrt{s_{\mathrm{NN}}}=5.02 \mathrm{TeV}$," Eur. Phys. J. C76 (2016) 245, arXiv: 1601.7868 [nucl-ex].

9. ALICE Collaboration, B. Abelev et al., " $\mathrm{K}^{*}(892)^{0}$ and $\phi(1020)$ production in $\mathrm{Pb}-\mathrm{Pb}$ collisions at $\sqrt{s_{\mathrm{NN}}}=2.76 \mathrm{TeV}$," Phys. Rev. C91 (2015) 024609, arXiv:1404.0495 [nucl-ex].

10. ALICE Collaboration, B. Abelev et al., "Production of $\Sigma(1385)^{ \pm}$and $\Xi(1530)^{0}$ in proton-proton collisions at $\sqrt{s}=7 \mathrm{TeV}, "$ Eur. Phys. J. C75 (2015) 1, arXiv:1406.3206 [nucl-ex].

11. ALICE Collaboration, D. Adamova et al., "Production of $\Sigma(1385)^{ \pm}$and $\Xi(1530)^{0}$ in $\mathrm{p}-\mathrm{Pb}$ collisions at $\sqrt{s_{\mathrm{NN}}}=5.02$ TeV," Eur. Phys. J. C77 (2017) 389, arXiv: 1701.07797 [nucl-ex].

12. J. Rafelski and B. Muller, "Strangeness Production in the Quark-Gluon Plasma," Phys. Rev. Lett. 48 (1982) 1066-1069. 


\section{E. Fragiacomo}

13. K. Redlich and A. Tounsi, "Strangeness enhancement and energy dependence in heavy ion collisions," Eur. Phys. J. C24 (2002) 589-594.

14. C. Blume and C. Markert, "Strange hadron production in heavy ion collisions from SPS to RHIC," Prog. Part. Nucl. Phys. 66 (2011) 834-879, arXiv:1105.2798 [nucl-ex].

15. S. Wheaton, J. Cleymans, and M. Hauer, "THERMUS - A thermal model package for ROOT," Comp. Phys. Comm. 180 (2009) 84-106.

16. T. Sjöstrand, S. Mrenna, and P. Skands, "A brief introduction to PYTHIA 8.1," Comput. Phys. Comm. 178 (2008) 852-867, arXiv:0710.3820 [hep-ph].

17. S. Roesler, R. Engel, , and J. Ranft, "The Monte Carlo Event Generator DPMJETIII, Advanced Monte Carlo for Radiation Physics, Particle Transport Simulation and Applications," Conference Proceedings, MC2000, Lisbon, Portugal, October 23-26 (2000) 1033-1038, hep-ph/0012252.

18. K. Werner, B. Guiot, I. Karpenko, and T. Pierog, "Analyzing radial flow features in $\mathrm{p}-\mathrm{Pb}$ and $\mathrm{p}-\mathrm{p}$ collisions at several $\mathrm{TeV}$ by studying identified-particle production with the event generator EPOS3," Phys. Rev. C 89 (2014) 064903.

19. C. Flensburg, G. Gustafsona, and L. Lönnblad, "Inclusive and exclusive observables from dipoles in high energy collisions", JHEP 08 (2011)103.

20. STAR Collaboration, B. I. Abelev et al., "Systematic measurements of identified particle spectra in pp, $\mathrm{d}-\mathrm{Au}$, and $\mathrm{Au}-\mathrm{Au}$ collisions at the STAR detector," Phys. Rev. C79 (2009) 034909.

21. ALICE Collaboration, B. Abelev et al., "Multi-strange baryon production in pp collisions at $\sqrt{s}=7 \mathrm{TeV}$ with ALICE," Phys. Lett. B712 (2012) 309, arXiv:1204.0282 [nucl-ex].

22. ALICE Collaboration, B. Abelev et al., "Production of $\Sigma(1385)^{ \pm}$and $\Xi(1530)^{0}$ in proton-proton collisions at $\sqrt{s}=7$ TeV," Eur. Phys. J. C75 (2015) 1, arXiv:1406.3206 [nucl-ex].

23. STAR Collaboration, B. I. Abelev et al., "Hadronic resonance production in $\mathrm{d}-\mathrm{Au}$ collisions at $\sqrt{s_{\mathrm{NN}}}=200 \mathrm{GeV}$ measured at the BNL Relativistic Heavy-Ion Collider," Phys. Rev. C78 (2008) 044906, arXiv:0801.0450 [nucl-ex].

24. ALICE Collaboration, J. Adam et al., "Multiplicity dependence of pion, kaon, proton and lambda production in $\mathrm{p}-\mathrm{Pb}$ collisions at $\sqrt{s_{\mathrm{NN}}}=5.02 \mathrm{TeV}$," Phys. Lett. B728 (2014) 25-38, arXiv:1307.6796 [nucl-ex].

25. ALICE Collaboration, J. Adam et al., "Multi-strange baryon production in $\mathrm{p}-\mathrm{Pb}$ collisions at $\sqrt{s_{\mathrm{NN}}}=5.02 \mathrm{TeV}$," Phys. Lett. B758 (2016) 389-401, arXiv: 1512.07227 [nucl-ex].

26. K. Aamodt et al. (ALICE Collaboration), "Two-pion Bose-Einstein correlations in central $\mathrm{Pb}-\mathrm{Pb}$ collisions at $\sqrt{s_{\mathrm{NN}}}=2.76 \mathrm{TeV}$ ", Phys. Lett. B696, (2011) 328.

27. Particle Data Group Collaboration, K. Olive et al., "Review of Particle Physics," Chin. Phys. C38 (2014) 090001.

28. M. Bleicher and J. Aichelin, "Strange resonance production: Probing chemical and thermal freezeout in relativistic heavy ion collisions," Phys. Lett. B. 530 (2002) 81-87, hep-ph/0201123.

29. A. Knospe, C. Markert, K. Werner, J. Steinheimer, and M. Bleicher, "Hadronic resonance production and interaction in partonic and hadronic matter in the EPOS3 model with and without the hadronic afterburner UrQMD," Phys. Rev. C 93 (2016) 014911. 